\title{
Convergence Analysis of Incomplete Biquadratic Rectangular Element for Fourth-Order Singular Perturbation Problem on Anisotropic Meshes
}

\author{
Pingli Xie ${ }^{1}$ and Meng $\mathrm{Hu}^{2}$ \\ ${ }^{1}$ School of Sciences, Henan University of Technology, Zhengzhou 450001, China \\ ${ }^{2}$ School of Mathematics and Statistics, Anyang Normal University, Anyang 455000, China \\ Correspondence should be addressed to Pingli Xie; xiexiepl@gmail.com
}

Received 2 June 2013; Accepted 29 December 2013; Published 13 February 2014

Academic Editor: Youyu Wang

Copyright (c) 2014 P. Xie and M. Hu. This is an open access article distributed under the Creative Commons Attribution License, which permits unrestricted use, distribution, and reproduction in any medium, provided the original work is properly cited.

The convergence analysis of a Morley type rectangular element for the fourth-order elliptic singular perturbation problem is considered. A counterexample is provided to show that the element is not uniformly convergent with respect to the perturbation parameter. A modified finite element approximation scheme is used to get convergent results; the corresponding error estimate is presented under anisotropic meshes. Numerical experiments are also carried out to demonstrate the theoretical analysis.

\section{Introduction}

The elliptic perturbation problems, which are derived from the stationary formation of parabolic perturbation problems, such as the Cahn-Hilliard type equation, are very important in both theoretical research and applications. The finite element methods are always chosen to be the appropriate way to solve the numerical solutions (cf. [1-5]). Here, we consider the following two-dimensional linear stationary Cahn-Hilliard type equation as our model problem:

$$
\begin{gathered}
\varepsilon^{2} \Delta^{2} u-\Delta u=f, \quad \text { in } \Omega, \\
u=\frac{\partial u}{\partial n}=0, \quad \text { on } \partial \Omega,
\end{gathered}
$$

where $\Delta$ is the standard Laplace operator, $\Omega$ is a bounded polygonal domain in $R^{2}, \partial \Omega$ is the boundary of $\Omega$, and $\varepsilon$ is a real parameter such that $0<\varepsilon \leq 1$. Let $\partial u / \partial n$ denote the normal derivative of $u$ along the boundary $\partial \Omega$. Particularly, the differential equations (1) formally degenerate to Poisson equations (a plate model degenerates towards an elastic membrane problem) when $\varepsilon$ tends to zero.

Semper considers its conforming finite element methods in [5]. The regularity of the solution is analyzed, quasioptimal global error estimates are presented when $\varepsilon>h$, and local analysis is also done by using techniques of Nitsche and Schatz [6] and Schatz and Wahlbin [7]. The author points out that the method behaves poorly when the perturbation parameter is much smaller than the mesh size by some numerical experiments.

On the other hand, it is well known that when fourthorder problems are discretized by a finite element method, the standard variational formulation will require the piecewise smooth functions in $C^{1}$ space. However, it is very difficult to construct such functions, and even if we can do that, the element will be rather complicated. Hence a common approach to solve this problem is to use nonconforming finite elements which violate the $C^{1}$-continuity requirement. In this case, two convergence criteria are generally employed: the Patch-Test [8] is used widely in engineers, but it is neither necessary nor sufficient; the Generalized Patch-Test [9] is proved to be the sufficient and necessary condition, while in practice it is often hard to be verified. To overcome the difficulty, the F-E-M criteria were proposed in [10] to make the test tractable.

Many successful nonconforming plate elements have been constructed (e.g., see $[2,3,9-17])$, but not all of them are convergent uniformly for (1) with respect to perturbation parameter $\varepsilon$. The very simple nonconforming Morley element 
(see [18]), which is convergent (cf. $[19,20])$ and even has some superconvergent properties under uniform meshes for plate problems, see [21], however, is proved to be not uniformly convergent for (1) in [3] when $\varepsilon \rightarrow 0$, that is; it may diverge for second order problem like Poisson equation (see also [22]). It is considered that the main reason for this degeneracy is the fact that the finite element space is not a subspace of $H^{1}(\Omega)$. Indeed, it is not of $C^{0}$ type. A counterexample is given in [3]. For more discussions on this element, we refer to $[3,19,22]$. As an alternative, a new modified $C^{0}$ element is proposed in [3], which is robust with respect to the parameter $\varepsilon$.

In [12], the convergence analysis of a nonconforming incomplete biquadratic rectangular plate element with the shape function space and the degrees of freedom $P(K)=\operatorname{span}\left\{1, x, y, x^{2}, x y, y^{2}, x^{2} y, x y^{2}\right\}$ and $\Sigma_{K}=$ $\left\{v_{i},(\partial v / \partial n)\left(B_{i}\right)(i=1,2,3,4)\right\}$, respectively, is studied, where $v_{i}$ is the function value at the vertex $a_{i}$ of element $K$, $(\partial v / \partial n)\left(B_{i}\right)$ is the unit outer normal derivative value at the middle point $B_{i}$ of the edge $l_{i}$ of $K$, and $n=\left(n_{x}, n_{y}\right)$ is the unit outer normal vector to $l_{i}$. This element, similar to the famous triangular Morley element $[3,18,19,23]$, is also a non $C^{0}$ element, and its convergence order $O(h)$ was given based on the Generalized Patch-Test. In [24], a modified element is provided by replacing the degrees of freedom and the shape function of [12] with $\Sigma_{K}=\left\{v_{i},\left(1 /\left|l_{i}\right|\right) \int_{l_{i}}(\partial v / \partial n) d s(i=\right.$ $1,2,3,4)\}$ and $P(K)=\operatorname{span}\left\{1, x, y, x^{2}, x y, y^{2}, x^{3}, y^{3}\right\}$, respectively. Recently, [2] applied the modified Morley element of [24] to the fourth-order elliptic singular perturbation problem and proved the convergence uniformly in the perturbation parameter. However, all the studies above are based on the traditional regular triangulations.

In this paper we will present another improved element by using the same degrees of freedom of [2] and the same shape function space of [12]. Obviously, the above element is convergent for fourth-order plate bending problems according to FEM test in [10]. However, to our knowledge, there is no literature considering the convergence of this element for fourth-order singular perturbation problems. Here, we will show that this element is not uniformly convergent for fourth-order singular perturbation problems with respect to the perturbation parameter $\varepsilon$ with a counterexample presented. Moreover, the convergence results are presented even under anisotropic meshes when the modified approximation formulation in [3] is employed.

The paper is organized as follows. The next section lists some preliminaries and the construction of the element. In Section 3, a counterexample is presented. In Section 4, the convergence results under the quasiuniform assumption and anisotropic meshes are provided. Numerical experiments are carried out in last section to confirm the theoretical analysis.

\section{Premilinaries}

Denote the inner product on $L^{2}(\Omega)$ by $(\cdot, \cdot)$, the usual Sobolev space, norm, and seminorm by $H^{m}(\Omega),\|\cdot\|_{m}$, and $|\cdot|_{m}$, respectively. The space $H_{0}^{m}(\Omega)$ is the closure in $H^{m}(\Omega)$ of $C_{0}^{\infty}(\Omega)$. Equivalently, we have

$$
\begin{gathered}
H_{0}^{1}(\Omega)=\left\{v \in H^{1}(\Omega) ;\left.v\right|_{\partial \Omega}=0\right\}, \\
H_{0}^{2}(\Omega)=\left\{v \in H^{2}(\Omega) ;\left.v\right|_{\partial \Omega}=\left.\frac{\partial v}{\partial n}\right|_{\partial \Omega}=0\right\} .
\end{gathered}
$$

Let $D u$ be the gradient of $u$ and let $D^{2} u=\left(\partial^{2} u / \partial x_{i} \partial x_{j}\right)_{2 \times 2}$ be the $2 \times 2$ tensor of the second order partial derivatives $(x=$ $\left.x_{1}, y=x_{2}\right)$. Define

$$
\begin{gathered}
a(u, v)=\int_{\Omega} D^{2} u: D^{2} v d x d y, \quad \forall u, v \in H^{2}(\Omega), \\
b(u, v)=\int_{\Omega} D u \cdot D v d x d y, \quad \forall u, v \in H^{1}(\Omega) .
\end{gathered}
$$

Then the weak form of (1) reads: find $u \in H_{0}^{2}(\Omega)$, such that

$$
\varepsilon^{2} a(u, v)+b(u, v)=(f, v), \quad \forall v \in H_{0}^{2}(\Omega) .
$$

By Green's formula, it is easy to get

$$
\int_{\Omega} D^{2} u: D^{2} v d x d y=\int_{\Omega} \Delta u \Delta v d x d y, \quad \forall u, v \in H_{0}^{2}(\Omega) .
$$

However, it does not hold on nonconforming finite element spaces.

Without loss of generality, we assume that the edges of $\Omega$ are parallel to the $x, y$ axis. For mesh size $h$, a rectangular triangulation $\mathscr{T}_{h}$ of $\Omega$ is then formed by lines also parallel to $x, y$ axis. Let $K \in \mathscr{T}_{h}$ be a rectangle with the central point $(0,0), 2 h_{x}$ and $2 h_{y}$ the lengths of edges parallel to $x$ axis and $y$ axis, respectively, $h_{K}=\max \left\{h_{x}, h_{y}\right\}, a_{1}\left(-h_{x},-h_{y}\right)$, $a_{2}\left(h_{x},-h_{y}\right), a_{3}\left(h_{x}, h_{y}\right)$, and $a_{4}\left(-h_{x}, h_{y}\right)$ the four vertices, $l_{i}=$ $\overrightarrow{a_{i} a_{i+1}}(i=1,2,3,4, \bmod (4))$. Let $\widehat{K}$ be a reference element in $(\xi, \eta)$ plane with central point $(0,0)$, four vertices $\widehat{a}_{1}(-1,-1)$, $\widehat{a}_{2}(1,-1), \widehat{a}_{3}(1,1)$, and $\widehat{a}_{4}(-1,1)$, and four edges $\widehat{l}_{i}=\overrightarrow{\hat{a}_{i} \widehat{a}_{i+1}}$, $(i=1,2,3,4, \bmod (4))$. Then there exists a reversible mapping $F_{K}: \widehat{K} \rightarrow K:$

$$
\begin{aligned}
& x=h_{x} \xi, \\
& y=h_{y} \eta .
\end{aligned}
$$

On $K$ we define the finite element $\left(K, P(K), \Sigma_{K}\right)$ as:

$$
\begin{gathered}
P(K)=P_{2}(K) \cup\left\{x^{2} y, x y^{2}\right\}, \\
\Sigma_{K}=\left\{v_{i}, \frac{1}{\left|l_{i}\right|} \int_{l_{i}} \frac{\partial v}{\partial n} d s,(i=1,2,3,4)\right\},
\end{gathered}
$$

where $P_{2}(K)$ denotes the set of quadratic polynomials on element $K$. Then it is easy to check that $P(K)$ can be uniquely determined by the degrees of freedom $\Sigma_{K}$. The degrees of freedom are plotted in Figure 1.

For every $v \in H^{3}(\Omega)$, we define the interpolation operator $\Pi_{h}$ as $\left.\Pi_{h} v\right|_{K}=\Pi_{K} v$, and $\Pi_{K}$ satisfies

$$
v \in H^{3}(K) \longrightarrow \Pi_{K} v \in P(K),
$$




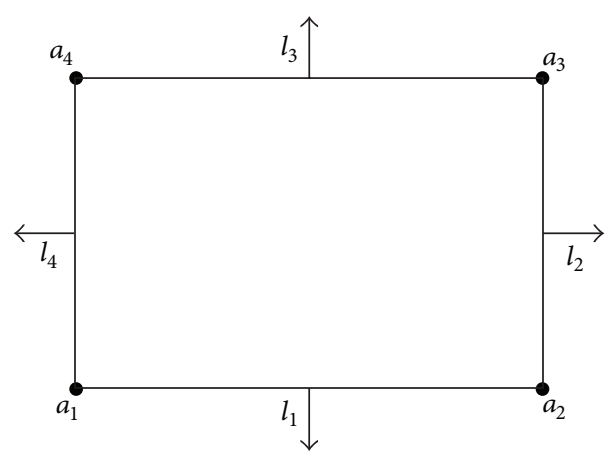

FIGURE 1: The degrees-of-freedom of incomplete biquadratic element.

such that

$$
\begin{gathered}
\Pi_{K} v\left(a_{i}\right)=v\left(a_{i}\right) \\
\frac{1}{\left|l_{i}\right|} \int_{l_{i}} \frac{\partial \Pi_{K} v}{\partial n} d s=\frac{1}{\left|l_{i}\right|} \int_{l_{i}} \frac{\partial v}{\partial n} d s, \quad(i=1,2,3,4) .
\end{gathered}
$$

It can be checked that

$$
\Pi_{K} v=v, \quad \forall v \in P_{2}(K)
$$

Let $V_{h}$ be the associated finite element space defined by

$$
\begin{aligned}
V_{h}= & \left\{v ;\left.v\right|_{K} \in P(K), \forall K \in \mathscr{T}_{h}, v(a)=0,\right. \\
& \left.\int_{l}\left[\frac{\partial v}{\partial n}\right] d s=0, \forall \text { node } a \in \partial \Omega, \forall l \subset \partial K\right\},
\end{aligned}
$$

where $[\partial v / \partial n]$ is the jump value of $\partial v / \partial n$ on $l \subset \partial K$ and $[\partial v / \partial n]=\partial v / \partial n$ if $l \subset \partial \Omega$.

Then the corresponding finite element approximation of (4) is as follows: find $u_{h} \in V_{h}$, such that

$$
\varepsilon^{2} a_{h}\left(u_{h}, v_{h}\right)+b_{h}\left(u_{h}, v_{h}\right)=\left(f, v_{h}\right), \quad \forall v_{h} \in V_{h},
$$

where for all $u_{h}, v_{h} \in V_{h}$,

$$
\begin{gathered}
a_{h}\left(u_{h}, v_{h}\right)=\sum_{T \in \mathscr{T}_{h}} \int_{T} D^{2} u_{h}: D^{2} v_{h} d x d y, \\
b_{h}\left(u_{h}, v_{h}\right)=\sum_{T \in \mathscr{T}_{h}} \int_{T} D u_{h} \cdot D v_{h} d x d y .
\end{gathered}
$$

Next, we will present the modified discretization form of problem (4) in [2].

Let $\Pi_{h}^{1}$ be the interpolation operator of the Lagrange bilinear rectangular element corresponding to the triangulation $\mathscr{T}_{h}$. The modified finite element method of (4) reads as: find $u_{h} \in V_{h}$, such that

$$
\varepsilon^{2} a_{h}\left(u_{h}, v_{h}\right)+b_{h}\left(\Pi_{h}^{1} u_{h}, \Pi_{h}^{1} v_{h}\right)=\left(f, \Pi_{h}^{1} v_{h}\right), \quad \forall v_{h} \in V_{h} .
$$

Note that the problem has a unique solution when $\varepsilon>0$, but when $\varepsilon=0$, the problem degenerates to

$$
b_{h}\left(\Pi_{h}^{1} u_{h}, \Pi_{h}^{1} v_{h}\right)=\left(f, \Pi_{h}^{1} v_{h}\right), \quad \forall v_{h} \in V_{h},
$$

in this case, $\Pi_{h}^{1} u_{h}$ is uniquely determined, though the solution $u_{h}$ is not unique.

We introduce the same mesh dependent norm $\|\cdot\|_{m, h}$ and semi-norm $|\cdot|_{m, h}$ on space $V_{h}+H^{m}$ :

$$
\begin{array}{r}
\|\cdot\|_{m, h}=\left(\sum_{K \in \mathscr{T}_{h}}\|\cdot\|_{m, K}^{2}\right)^{1 / 2}, \quad|\cdot|_{m, h}=\left(\sum_{K \in \mathscr{T}_{h}}|\cdot|_{m, K}^{2}\right)^{1 / 2}, \\
m=0,1,2,3 .
\end{array}
$$

The energy norm is defined by

$$
\left\|\left|v_{h}\right|\right\|_{\varepsilon, h}=\varepsilon^{2} a_{h}\left(v_{h}, v_{h}\right)+b_{h}\left(\Pi_{h}^{1} v_{h}, \Pi_{h}^{1} v_{h}\right) .
$$

\section{A Counterexample}

In this section, we construct a counterexample to show that the element presented in Section 2 is not convergent uniformly with respect to the perturbation parameter $\varepsilon$. That means if the element is applied to a nearly second order problem with the form of (1) when $\varepsilon \rightarrow 0$, the convergence rate of the method will deteriorate. In fact, like the Morley element, when it is applied to a second order equation like Poisson's equation, the method will diverge.

As in [3], we consider the slightly modified reduced problem

$$
\begin{gathered}
-\Delta u=f, \quad \text { in } \Omega, \\
u=0, \quad \text { on } \Gamma_{D}, \\
\frac{\partial u}{\partial n}=g, \quad \text { on } \Gamma_{N},
\end{gathered}
$$

to simplify some calculations. Assume $\partial \Omega=\Gamma_{D} \cup \Gamma_{N}$, where $\Gamma_{N}$ and $\Gamma_{D}$ are disjoint subsets of $\partial \Omega$. This problem is a second order problem with mixed boundary conditions, which can be regarded as the formal limit of the fourth-order problems

$$
\begin{gathered}
\varepsilon^{2} \Delta^{2} u-\Delta u=f, \quad \text { in } \Omega, \\
u=0, \quad \text { on } \Gamma_{D}, \\
\frac{\partial}{\partial n}\left(u-\varepsilon^{2} \Delta u\right)=g, \quad \text { on } \Gamma_{N}, \\
\Delta u=0, \quad \text { on } \partial \Omega .
\end{gathered}
$$

Let $\mathscr{T}_{h}$ be a triangulation of $\Omega$, and let $\widetilde{V}_{h}$ be the finite element space of incomplete biquadratic plate element corresponding to the boundary conditions of (18) here; see Figure 2. Then the approximation problem to (18) reads as: find $u_{h} \in \widetilde{V}_{h}$, such that

$$
b_{h}\left(u_{h}, v_{h}\right)=\left(f, v_{h}\right)+\left\langle g, v_{h}\right\rangle, \quad \forall v_{h} \in \widetilde{V}_{h},
$$




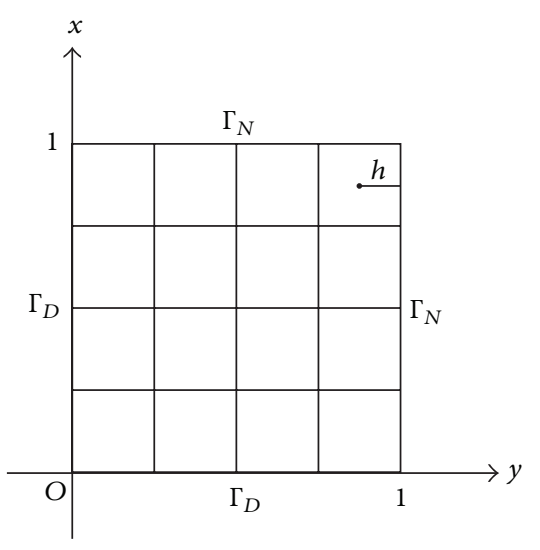

Figure 2: The triangulation of $\Omega$.

where $\left\langle g, v_{h}\right\rangle=\int_{\Gamma_{N}} g v_{h} d s, d s$ denotes the arc length along $\Gamma_{N}$. Moreover, the exact solution $u$ of (18) satisfies

$$
b_{h}\left(u, v_{h}\right)=\left(f, v_{h}\right)+\left\langle g, v_{h}\right\rangle+E_{h}\left(u, v_{h}\right), \quad \forall v_{h} \in \widetilde{V}_{h},
$$

where

$$
E_{h}\left(u, v_{h}\right)=b_{h}\left(u, v_{h}\right)-\left(f, v_{h}\right)-\left\langle g, v_{h}\right\rangle .
$$

Let $\||\cdot|\|_{h}$ be the corresponding energy norm of problem (18), that is, $\||\cdot|\|_{h}=b_{h}(\cdot, \cdot)$. Then, employing Cauchy-Schwarz inequality (we refer to [3]), we have

$$
\left\|\left|u-u_{h}\right|\right\|_{h} \geq \sup _{v_{h} \in \widetilde{V}_{h}} \frac{\left|E_{h}\left(u, v_{h}\right)\right|}{\left\|\left|v_{h}\right|\right\|_{h}} .
$$

In the following, we will choose a suitable exact solution $u$ to prove the divergence of the method by virtue of (23).

The domain $\Omega$ is taken as the unit square. To simplify the analytic process, the uniform triangulation with the mesh size $1 / n$ is employed. Assume $\Gamma_{D}$ to be the intersection of $\partial \Omega$ with the coordinate axis, while $\Gamma_{N}$ to be the part of $\partial \Omega$ on $x=1$ and $y=1$. Hence, the functions in finite element space $\widetilde{V}_{h}$ are zeros at the vertices on the coordinate axis.

We assume that the exact solution of (18) is given by $u=$ $x y$. Thus, $g=x$ on $y=1$ and $g=y$ on $x=1$. Obviously, $u$ is harmonic, which means $f=0$. Therefore:

$$
E_{h}\left(u, v_{h}\right)=b_{h}\left(u, v_{h}\right)-\left\langle g, v_{h}\right\rangle .
$$

Note that $u \in \widetilde{V}_{h}$, thus $\Pi_{h} u=u$ and here $\Pi_{h}$ is the finite element interpolation on $\widetilde{V}_{h}$. Similar to the discussion in [3], it is easy to derive that finite element space $\widetilde{V}_{h}$ can be naturally decomposed into two spaces:

$$
\widetilde{V}_{h}=\widetilde{V}_{h}^{v}+\widetilde{V}_{h}^{e},
$$

where $\widetilde{V}_{h}^{v}$ and $\widetilde{V}_{h}^{e}$ correspond to the vertex values and the edge values, respectively. In fact, the two spaces can be expressed as

$$
\begin{gathered}
\widetilde{V}_{h}^{v}=\left\{v_{h} \in \widetilde{V}_{h}: \int_{e} \frac{\partial v_{h}}{\partial n} d s=0, \forall e \in \mathscr{E}_{h}\right\}, \\
\widetilde{V}_{h}^{e}=\left\{v_{h} \in \widetilde{V}_{h}: v_{h}(x)=0, \forall x \in \mathscr{X}_{h}\right\},
\end{gathered}
$$

where $\mathscr{E}_{h}$ and $\mathscr{X}_{h}$ represent the sets of the edges and vertices corresponding to the triangulation $\mathscr{T}_{h}$, respectively. Let $u_{h}^{v}$ be the interpolant of $u$ onto $\widetilde{V}_{h}^{v}$, then, according to its definition, we can get

$$
\begin{gathered}
u_{h}^{v}(x)=u(x), \quad \forall x \in \mathscr{X}_{h}, \\
\int_{e} \frac{\partial u_{h}^{v}}{\partial n} d s=0, \quad \forall e \in \mathscr{E}_{h} .
\end{gathered}
$$

We begin to prove that the $\operatorname{limit}_{\lim _{h \rightarrow 0}}\left(\left|E_{h}\left(u, v_{h}\right)\right| /\right.$ $\left.\left\|\left|v_{h}\right|\right\|_{h}\right)$ is strictly positive, so we can show from (23) that the method is divergent.

In [3], the authors proved for Morley element, the decomposition of space $\widetilde{V}_{h}$ is orthogonal, while it does not hold any more for this incomplete biquadratic plate element. However, in the mesh fashion chosen before, for any element $K \in \mathscr{T}_{h}$, the length of each edge is $2 h=1 / n$. Let the center point of $K$ be $\left(x_{0}, y_{0}\right)$, then by the definition of the space $\widetilde{V}_{h}^{v}$, direct calculation implies the expression of $u_{h}^{v}$ on element $K$ :

$$
u_{h}^{v}=x_{0} y_{0}+\frac{1}{2} \eta\left(3 \xi^{2}-1\right) x_{0} h+\frac{1}{2} \xi\left(3 \eta^{2}-1\right) y_{0} h+\xi \eta h^{2}
$$

where

$$
\xi=\frac{\left(x-x_{0}\right)}{h}, \quad \eta=\frac{\left(y-y_{0}\right)}{h} .
$$

We denote the element $[-1,1] \times[-1,1]$ on $(\xi, \eta)$ plane by the reference element $\widehat{K}$. Apparently, the mapping from $\widehat{K}$ to $K$ is affine, hence we have

$$
\begin{aligned}
& \frac{\partial u_{h}^{v}}{\partial x}=3 \xi \eta x_{0}+\frac{1}{2}\left(3 \eta^{2}-1\right) y_{0}+\eta h, \\
& \frac{\partial u_{h}^{v}}{\partial y}=3 \xi \eta y_{0}+\frac{1}{2}\left(3 \xi^{2}-1\right) x_{0}+\xi h,
\end{aligned}
$$

moreover, $\partial u / \partial x=y, \partial u / \partial y=x$, and then

$$
\begin{array}{rl}
\int_{K} & D u \cdot D u_{h}^{v} d x d y \\
= & \int_{K} \frac{\partial u}{\partial x} \frac{\partial u_{h}^{v}}{\partial x}+\frac{\partial u}{\partial y} \frac{\partial u_{h}^{v}}{\partial y} d x d y \\
= & h^{2} \int_{\widehat{K}}\left(h \eta+y_{0}\right)\left(3 \xi \eta x_{0}+\frac{1}{2}\left(3 \eta^{2}-1\right) y_{0}+\eta h\right) \\
& +\left(h \xi+x_{0}\right)\left(3 \xi \eta y_{0}+\frac{1}{2}\left(3 \xi^{2}-1\right) x_{0}+\xi h\right) d \xi d \eta \\
= & h^{2} \int_{-1}^{1} \int_{-1}^{1}\left(h^{2} \eta^{2}+h^{2} \xi^{2}\right) d \xi d \eta \\
= & \frac{4}{3} h^{4},
\end{array}
$$

thus

$$
\lim _{h \rightarrow 0} b_{h}\left(u, u_{h}^{v}\right)=\lim _{h \rightarrow 0} \sum_{K \in \mathscr{T}_{h}} \frac{4}{3} h^{4}=\lim _{h \rightarrow 0} n^{2} \frac{4}{3} h^{4}=\lim _{h \rightarrow 0} \frac{1}{3} h^{2}=0 .
$$


On the other hand, denoting the edges of $\Omega$ on $y=1$ and $x=1$ by $\Gamma_{N_{1}}$ and $\Gamma_{N_{2}}$, respectively, we have

$$
\left\langle g, u_{h}^{v}\right\rangle=\int_{\Gamma_{N}} g u_{h}^{v} d s=\int_{\Gamma_{N_{1}}} g u_{h}^{v} d s+\int_{\Gamma_{N_{2}}} g u_{h}^{v} d s .
$$

We first consider the first term, let those components of $\mathscr{E}_{h}$ lying on $\Gamma_{N_{1}}$ be $l_{i}, i=1,2, \ldots, n$, and let the corresponding element be $K_{i}$, the center point of $K_{i}$ be $\left(x_{i}, y_{*}\right)$, where $x_{i}=$ $(2 i-1) / 2 n=h(2 i-1), y_{*}=(2 n-1) / 2 n=1-h$. Then

$$
\begin{aligned}
& \int_{l_{i}} g u_{h}^{v} d s \\
& =h \int_{\hat{l}_{i}}\left(h \xi+x_{i}\right)\left(x_{i} y_{*}+\frac{1}{2} \eta\left(3 \xi^{2}-1\right) x_{i} h\right. \\
& \left.\quad+\frac{1}{2} \xi\left(3 \eta^{2}-1\right) y_{*} h+\xi \eta h^{2}\right) d \widehat{s} \\
& =h \int_{-1}^{1}\left(h \xi+x_{i}\right) \quad \\
& \quad \times\left(x_{i} y_{*}+\frac{1}{2} \eta\left(3 \xi^{2}-1\right) x_{i} h+\xi y_{*} h+\xi h^{2}\right) d \xi \\
& =2 h\left(\frac{1}{3} y_{*} h^{2}+\frac{1}{3} h^{3}+x_{i}^{2} y_{*}\right),
\end{aligned}
$$

thus

$$
\begin{aligned}
\int_{\Gamma_{N_{1}}} g u_{h}^{v} d s= & \sum_{i=1}^{n} 2 h\left(\frac{1}{3}(1-h) h^{2}+\frac{1}{3} h^{3}\right. \\
& \left.\quad+\left(\frac{2 i-1}{2 n}\right)^{2}(1-h)\right) \\
= & \sum_{i=1}^{n} 2 h\left(\frac{1}{3}(1-h) h^{2}+\frac{1}{3} h^{3}\right. \\
= & \left.\frac{1}{3} h^{2}+2 h^{2}(2 i-1)^{2}(1-h)\right) \\
& \times\left(\frac{(2 h+1)(h+1)}{6 h^{3}}-\frac{2 h+1}{2 h^{2}}+\frac{1}{2 h}\right) .
\end{aligned}
$$

Therefore, by a limit process, we can obtain

$$
\lim _{h \rightarrow 0} \int_{\Gamma_{N_{1}}} g u_{h}^{v} d s=\frac{1}{3} .
$$

Similarly, for $\int_{\Gamma_{N_{2}}} g u_{h}^{v} d s$, we have

$$
\begin{aligned}
& \int_{\Gamma_{N_{2}}} g u_{h}^{v} d s \\
& =\sum_{i=1}^{n} 2 h\left(\frac{1}{3}(1-h) h^{2}+\frac{1}{3} h^{3}+\left(\frac{2 i-1}{2 n}\right)^{2}(1-h)\right) \\
& =\int_{\Gamma_{N_{1}}} g u_{h}^{v} d s
\end{aligned}
$$

this immediately leads to

$$
\lim _{h \rightarrow 0} \int_{\Gamma_{N_{2}}} g u_{h}^{v} d s=\frac{1}{3} .
$$

Finally, since $\left\|\left|u_{h}^{v}\right|\right\|_{h}^{2}=b_{h}\left(u_{h}^{v}, u_{h}^{v}\right)=$ $\sum_{K \in \mathscr{T}_{h}} \int_{K}\left|D u_{h}^{v}\right|^{2} d x d y$, and

$$
\begin{aligned}
\int_{K}\left|D u_{h}^{v}\right|^{2} d x d y= & \int_{K}\left(\frac{\partial u_{h}^{v}}{\partial x}\right)^{2}+\left(\frac{\partial u_{h}^{v}}{\partial y}\right)^{2} d x d y \\
= & h^{2} \int_{\widehat{K}}\left(3 \xi \eta x_{0}+\frac{1}{2}\left(3 \eta^{2}-1\right) y_{0}+\eta h\right)^{2} \\
& +\left(3 \xi \eta y_{0}+\frac{1}{2}\left(3 \xi^{2}-1\right) x_{0}+\xi h\right)^{2} d \xi d \eta \\
= & \frac{24}{5} h^{2}\left(x_{0}^{2}+y_{0}^{2}\right)+\frac{8}{3} h^{4},
\end{aligned}
$$

we can verify that

$$
\begin{aligned}
b_{h}\left(u_{h}^{v}, u_{h}^{v}\right) & =\sum_{K \in \mathscr{T}_{h}}\left(\frac{24}{5} h^{2}\left(x_{0}^{2}+y_{0}^{2}\right)+\frac{8}{3} h^{4}\right) \\
& =\frac{24}{5} h^{4} 2 n \sum_{i=1}^{n}(2 i-1)^{2}+\frac{2}{3} h^{2} \\
& =\frac{4}{5}\left(1+2 h^{2}\right)+\frac{2}{3} h^{2} .
\end{aligned}
$$

From this expression we obtain that

$$
\lim _{h \rightarrow 0} b_{h}\left(u_{h}^{v}, u_{h}^{v}\right)=\frac{4}{5} .
$$

This together with (32), (36), and (38) implies

$$
\lim _{h \rightarrow 0} \frac{\left|E_{h}\left(u, u_{h}^{v}\right)\right|}{\left\|\left|u_{h}^{v}\right|\right\|_{h}}=\lim _{h \rightarrow 0} \frac{\left|b_{h}\left(u, u_{h}^{v}\right)-\left\langle g, u_{h}^{v}\right\rangle\right|}{b_{h}\left(u_{h}^{v}, u_{h}^{v}\right)^{1 / 2}}=\frac{2 / 3}{\sqrt{4 / 5}}=\frac{\sqrt{5}}{3} .
$$

The divergence of the method is therefore a consequence of the basic lower bound (23).

\section{Convergence Analysis in a Modified Discretization Form}

In the last section, we provide a counterexample to show that the incomplete biquadratic plate element may diverge for a second order problem like Poisson equation, and hence, in the standard finite element approximation, the convergence can not be insured for problem (1). In [2], a new modified approximation form is presented for Morley element and another Morley type rectangular element. In this section, we will show that the incomplete biquadratic plate element is convergent for problem (1) uniformly with respect to the parameter $\varepsilon$ under anisotropic meshes.

To begin with, we introduce the following error estimate regarding the operator $\Pi_{h}^{1}$ on anisotropic meshes, as to its proof, we refer to [25]. 
Lemma 1. For the bilinear interpolation operator $\Pi_{h}^{1}$, for all $u \in H^{2}(\Omega)$, there holds

$$
\left\|u-\Pi_{h}^{1} u\right\|_{0, \Omega}+h\left\|\nabla\left(u-\Pi_{h}^{1} u\right)\right\|_{0, \Omega} \leq C h^{2}|u|_{2, \Omega},
$$

where $C$ is a constant independent of triangulation.

Denote the quadratic part of the interpolant function $\widehat{\Pi} \widehat{v}$ by $\overline{\widehat{\Pi}} \widehat{v}$ and the corresponding part of the function $\Pi_{K} v$ on the element $K$ by $\overline{\Pi_{K} v}$, then we can get the following lemma.

Lemma 2. For any $K \in \mathscr{T}_{h}$, for all $v \in H^{2}(K)$, without the regular or quasiuniform assumption, we have the following estimate:

$$
\left|v-\Pi_{K}^{1} \overline{\Pi_{K} v}\right|_{1, K} \leq C h|v|_{2, K}, \quad \forall v \in H^{2}(K) .
$$

Proof. Apparently, $\Pi_{K}^{1} \overline{\Pi_{K} v}$ can be considered as an interpolant of $v$ on $Q_{1}(K)$. For simplicity, we denote it by $\widetilde{\Pi} v$. We first show that on the reference element $\widehat{K}$, for multi-index $|\alpha|=1$,

$$
\left\|\widehat{D}^{\alpha}(\widehat{v}-\widehat{\widetilde{\Pi}} \widehat{v})\right\|_{0, \widehat{K}} \leq C\left|\widehat{D}^{\alpha} \widehat{v}\right|_{1, \widehat{K}} .
$$

For the convenience of notations, we denote degrees-offreedom by $v\left(a_{i}\right)=v_{i},\left(1 /\left|l_{i}\right|\right) \int_{l_{i}}(\partial v / \partial n) d s=v_{i+4}, i=1,2,3,4$. Correspondingly, $\widehat{v}\left(\widehat{a}_{i}\right)=\widehat{v}_{i},\left(1 /\left|\widehat{l}_{i}\right|\right) \int_{\widehat{l}_{i}}(\partial \widehat{v} / \partial n) d s=\widehat{v}_{i+4}$, $i=1,2,3,4$. Hence, for all $\widehat{v} \in H^{1}(\widehat{K})$, direct computation provides the expression of $\widehat{\widetilde{\Pi}} \widehat{v}$ :

$$
\widehat{\widetilde{\Pi}} \widehat{v}=\beta_{0}+\beta_{1} \xi+\beta_{2} \eta+\beta_{3} \xi \eta
$$

where

$$
\begin{aligned}
& \beta_{0}=\frac{1}{4}\left(\widehat{v}_{1}+\widehat{v}_{2}+\widehat{v}_{3}+\widehat{v}_{4}\right), \\
& \beta_{1}=\frac{1}{8}\left(\widehat{v}_{1}-\widehat{v}_{2}-\widehat{v}_{3}+\widehat{v}_{4}\right)+\frac{3}{4}\left(\widehat{v}_{6}-\widehat{v}_{8}\right), \\
& \beta_{2}=\frac{1}{8}\left(\widehat{v}_{1}+\widehat{v}_{2}-\widehat{v}_{3}+\widehat{v}_{4}\right)-\frac{3}{4}\left(\widehat{v}_{5}-\widehat{v}_{7}\right), \\
& \beta_{3}=\frac{1}{4}\left(\widehat{v}_{1}-\widehat{v}_{2}+\widehat{v}_{3}-\widehat{v}_{4}\right),
\end{aligned}
$$

thus, when $\alpha=(1,0)$, we have

$$
\widehat{D}^{\alpha}(\widehat{\widetilde{\Pi}} \widehat{v})=\beta_{1}+\beta_{3} \eta \text {. }
$$

Obviously, $\{1, \eta\}$ is a basis of $\widehat{D}^{\alpha} \widehat{Q}_{1}$. Moreover, let $\widehat{w}=\partial \widehat{v} / \partial \xi$, we can get

$$
\begin{aligned}
\beta_{1}= & \frac{1}{8}\left(\widehat{v}_{1}-\widehat{v}_{2}-\widehat{v}_{3}+\widehat{v}_{4}\right)+\frac{3}{4}\left(\widehat{v}_{6}-\widehat{v}_{8}\right) \\
= & -\frac{1}{8} \int_{-1}^{1}\left(\frac{\partial \widehat{v}}{\partial \xi}(\xi,-1)+\frac{\partial \widehat{v}}{\partial \xi}(\xi, 1)\right) d \xi \\
& +\frac{3}{8} \int_{-1}^{1}\left(\frac{\partial \widehat{v}}{\partial \xi}(1, \eta)+\frac{\partial \widehat{v}}{\partial \xi}(-1, \eta)\right) d \eta
\end{aligned}
$$

$$
\begin{aligned}
= & -\frac{1}{8} \int_{-1}^{1} \int_{-1}^{1} \frac{\partial}{\partial \eta}\left(\eta \frac{\partial \widehat{v}}{\partial \xi}\right) d \xi d \eta \\
& +\frac{3}{8} \int_{-1}^{1} \int_{-1}^{1} \frac{\partial}{\partial \xi}\left(\xi \frac{\partial \widehat{v}}{\partial \xi}\right) d \xi d \eta \\
= & : F_{1}(\widehat{w}), \\
\beta_{3}= & \frac{1}{4}\left(\widehat{v}_{1}-\widehat{v}_{2}+\widehat{v}_{3}-\widehat{v}_{4}\right) \\
= & \frac{1}{4} \int_{-1}^{1}\left(\frac{\partial \widehat{v}}{\partial \xi}(\xi, 1)-\frac{\partial \widehat{v}}{\partial \xi}(\xi,-1)\right) d \xi \\
= & \frac{1}{4} \int_{-1}^{1} \int_{-1}^{1} \frac{\partial^{2} \widehat{v}}{\partial \xi \partial \eta} d \xi d \eta \\
= & : F_{2}(\widehat{w}) .
\end{aligned}
$$

By Hölder's inequality, $F_{j}(\widehat{w})(j=1,2)$ is a bounded linear functional on $H^{1}(\widehat{K})$. Therefore, by the basic theorem in [26], (45) holds. Then,

$$
\begin{aligned}
\left\|(v-\widetilde{\Pi} v)_{x}\right\|_{0, K}^{2} & =h_{x}^{-2} h_{x} h_{y}\left\|(\widehat{v}-\widehat{\widetilde{\Pi}} \widehat{v})_{\xi}\right\|_{0, \widehat{K}}^{2} \\
& \leq C h_{x}^{-2} h_{x} h_{y}\left|\widehat{v}_{\xi}\right|_{1, \widehat{K}}^{2} \\
& =C h_{x}^{-2} h_{x} h_{y}\left(\left|\widehat{v}_{\xi \xi}\right|_{0, \widehat{K}}^{2}+\left|\widehat{v}_{\xi \eta}\right|_{0, \widehat{K}}^{2}\right) \\
& =C h_{x}^{-2}\left(h_{x}^{4}\left|v_{x x}\right|_{0, K}^{2}+h_{x}^{2} h_{y}^{2}\left|v_{x y}\right|_{0, K}^{2}\right) \\
& \leq C h^{2}|v|_{2, K}^{2} .
\end{aligned}
$$

The conclusion when $\alpha=(0,1)$ can be derived similarly, that is,

$$
\left\|(v-\widetilde{\Pi} v)_{y}\right\|_{0, K}^{2} \leq C h^{2}|v|_{2, K}^{2} .
$$

Equations (50) and (51) immediately imply the desired result.

The following error estimates can be found in [27].

Lemma 3. For all $u \in H^{3}(\Omega)$, without the regular or quasiuniform assumption, the following estimates hold:

$$
\begin{gathered}
\left\|u-\overline{\Pi_{h} u}\right\|_{h} \leq \frac{4 h}{\pi}|u|_{3}, \\
\left|\sum_{K \in \mathscr{T}_{h}} \int_{\partial K}\left(\Delta u-\frac{\partial^{2} u}{\partial s^{2}}\right) \frac{\partial v_{h}}{\partial n} d s\right| \leq C h|u|_{3}\left|v_{h}\right|_{2, h}, \quad \forall v_{h} \in V_{h}, \\
\left|\sum_{K \in \mathscr{T}_{h}} \int_{\partial K} \frac{\partial^{2} u}{\partial s \partial n} \frac{\partial v_{h}}{\partial s} d s\right| \leq C h|u|_{3}\left|v_{h}\right|_{2, h}, \quad \forall v_{h} \in V_{h} .
\end{gathered}
$$

The following theorem shows that for any fixed $\varepsilon \in(0,1]$ the new incomplete biquadratic element method converges linearly with respect to $h$. 
Theorem 4. Suppose $u$ and $u_{h}$ are the solutions of (1) and (14), respectively, $u \in H^{3}(\Omega)$, then without the quasiuniform assumption and regular condition, there exists a constant $C$ independent of $h$ and $\varepsilon$, such that

$$
\left\|\left|u-u_{h}\right|\right\|_{\varepsilon, h} \leq \operatorname{Ch}\left(\varepsilon|u|_{3, \Omega}+|u|_{2, \Omega}\right) .
$$

Proof. The second Strang lemma implies

$$
\left\|\left|u-u_{h}\right|\right\|_{\varepsilon, h} \leq C\left(\inf _{v_{h} \in V_{h}}\left\|\left|u-u_{h}\right|\right\|_{\varepsilon, h}+\sup _{w_{h} \in V_{h}} \frac{\left|E_{\varepsilon, h}\left(u, w_{h}\right)\right|}{\left\|\left|w_{h}\right|\right\|_{\varepsilon, h}}\right),
$$

where $E_{\varepsilon, h}\left(u, w_{h}\right)$ is the consistency error given by

$$
E_{\varepsilon, h}\left(u, w_{h}\right)=\varepsilon^{2} a_{h}\left(u, w_{h}\right)+b\left(\Pi_{h}^{1} u, \Pi_{h}^{1} w_{h}\right)-f\left(\Pi_{h}^{1} w_{h}\right) .
$$

Furthermore, from Lemmas 1 and 2,

$$
\begin{aligned}
& \inf _{v_{h} \in V_{h}}\left\|\left|u-u_{h}\right|\right\|_{\varepsilon, h} \\
& \quad \leq \|\left.\left|u-\overline{\Pi_{h} u}\right|\right|_{\varepsilon, h} \\
& \quad=\left(\varepsilon^{2}\left|u-\overline{\Pi_{h} u}\right|_{2, h}^{2}+\left|\Pi_{h}^{1} u-\Pi_{h}^{1} \overline{\Pi_{h} u}\right|_{1, h}^{2}\right)^{1 / 2} \\
& \quad \leq \operatorname{Ch}\left(\varepsilon|u|_{3}+|u|_{2}\right) .
\end{aligned}
$$

Hence, it suffices to estimate the consistency error $E_{\varepsilon, h}\left(u, w_{h}\right)$.

Since $u \in H^{3}$ and $\Pi_{h}^{1} w_{h}$ are continuous, it follows from (1) that

$$
\begin{aligned}
\left(f, \Pi_{h}^{1} w_{h}\right)=\int_{\omega}( & \left.-\varepsilon^{2} D(\Delta u)+D u\right) \\
\cdot & D\left(\Pi_{h}^{1} w_{h}\right) d x d y, \quad \forall w_{h} \in V_{h} .
\end{aligned}
$$

By using the approximation formulation, the consistency error can therefore be expressed as

$$
\begin{aligned}
E_{\varepsilon, h}\left(u, w_{h}\right) & \\
= & \varepsilon^{2} \sum_{K \in \mathscr{T}_{h}} \int_{K}\left(D^{2} u: D^{2} w_{h}+D(\Delta u) \cdot D \Pi_{h}^{1} w_{h}\right) d x d y \\
& +\sum_{K \in \mathscr{T}_{h}} \int_{K}\left(D \Pi_{h}^{1} u-D u\right) \cdot D \Pi_{h}^{1} w_{h} d x d y
\end{aligned}
$$

On the other hand,

$$
D^{2} u: D^{2} w_{h}=\Delta u \Delta w_{h}+\left(2 u_{x y} v_{x y}-u_{x x} v_{y y}-u_{y y} v_{x x}\right),
$$

and by Green's formula, $E_{\varepsilon, h}\left(u, w_{h}\right)$ can be rewritten as

$$
\begin{aligned}
E_{\varepsilon, h}\left(u, w_{h}\right)= & \varepsilon^{2} \sum_{K \in \mathscr{T}_{h}} \int_{K} D(\Delta u) \cdot D\left(\Pi_{h}^{1} w_{h}-w_{h}\right) d x d y \\
& +\sum_{K \in \mathscr{T}_{h}} \int_{K} D\left(\Pi_{h}^{1} u-u\right) \cdot D \Pi_{h}^{1} w_{h} d x d y
\end{aligned}
$$

$$
\begin{aligned}
& +\varepsilon^{2} \sum_{K \in \mathscr{T}_{h}} \int_{\partial K}\left(\Delta u-\frac{\partial^{2} u}{\partial s^{2}}\right) \frac{\partial v_{h}}{\partial n} d s \\
& +\varepsilon^{2} \sum_{K \in \mathscr{T}_{h}} \int_{\partial K} \frac{\partial^{2} u}{\partial s \partial n} \frac{\partial v_{h}}{\partial s} d s .
\end{aligned}
$$

It follows from Hölder's inequality and Lemma 1 that

$$
\begin{gathered}
\sum_{K \in \mathscr{T}_{h}} \int_{K} D(\Delta u) \cdot D\left(\Pi_{h}^{1} w_{h}-w_{h}\right) d x d y \leq C h|u|_{3}\left|w_{h}\right|_{2, h}, \\
\sum_{K \in \mathscr{T}_{h}} \int_{K} D\left(\Pi_{h}^{1} u-u\right) \cdot D \Pi_{h}^{1} w_{h} d x d y \leq C h|u|_{3}\left|\Pi_{h}^{1} w_{h}\right|_{1, h}
\end{gathered}
$$

Together with Lemma 3, we can immediately get the desired estimate.

Remark 5. By Lemma 1 and the estimates above, we can derive the same convergence result as in [2]:

$$
\varepsilon\left|u-u_{h}\right|_{2, h}+\left|u-\Pi_{h}^{1} u_{h}\right|_{1, h} \leq \operatorname{Ch}\left(\varepsilon|u|_{3, \Omega}+|u|_{2, \Omega}\right) .
$$

We should mention that the result here does not need the quasiuniform assumptions. Moreover, similar discussions can also lead to the following estimate when the meshes satisfy the quasiuniform assumption

$$
\varepsilon\left|u-u_{h}\right|_{2, h}+\left|u-\Pi_{h}^{1} u_{h}\right|_{1, h} \leq C h^{1 / 2}\|f\|_{0, \Omega} .
$$

Remark 6. In the last section, a counterexample is presented to show the possible divergence of incomplete biquadratic plate element when applied to second order problem, but the theorem above implicates that, when the approximation formulation (14) is employed, the uniform convergence result can be ensured even without the regular condition or quasiuniform assumption.

\section{Numerical Experiments}

In this section, numerical experiments are carried out to confirm our theoretical analysis of the incomplete biquadratic element. We calculate several numerical examples for problem (1) in different approximation schemes. We consider problem (1) with $\Omega=[0,1]^{2} \subset R^{2}$ and $f=\varepsilon^{2} \Delta^{2} u-\Delta u$, where $u=(\sin \pi x \sin \pi y)^{2}$. The domain $\Omega$ is divided into the following two fashions.

Mesh 1: square mesh. The mesh obtained in this way for $n=16$ is illustrated in Figure 3(a).

Mesh 2: each edge of $\Omega$ is divided into $n$ segments with $n+1$ points $\sin (\pi i / n) / 2, i=0,1, \ldots, n / 2,1-$ $\sin (\pi i / n) / 2, i=n / 2+1, \ldots, n$. The mesh obtained in this way for $n=16$ is illustrated in Figure 3(b).

We first compute the relative errors in the energy norm $\left\|\left|u-u_{h}\right|\right\|_{\varepsilon, h} /\||u|\|_{\varepsilon, h}$ under mesh 1 when we use the standard 


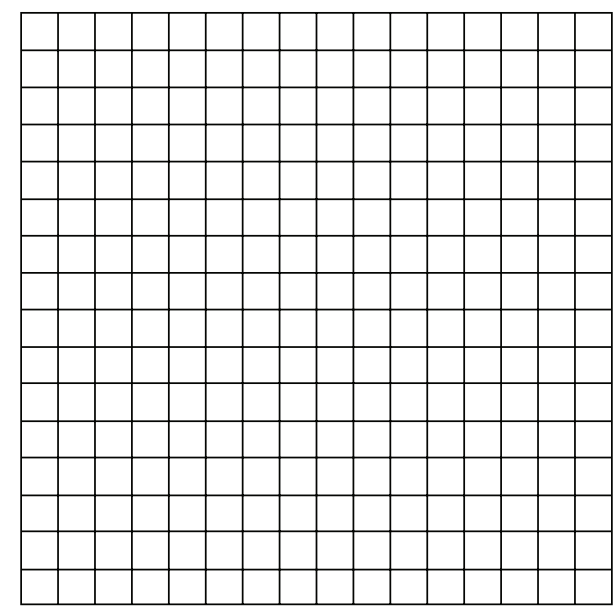

(a)

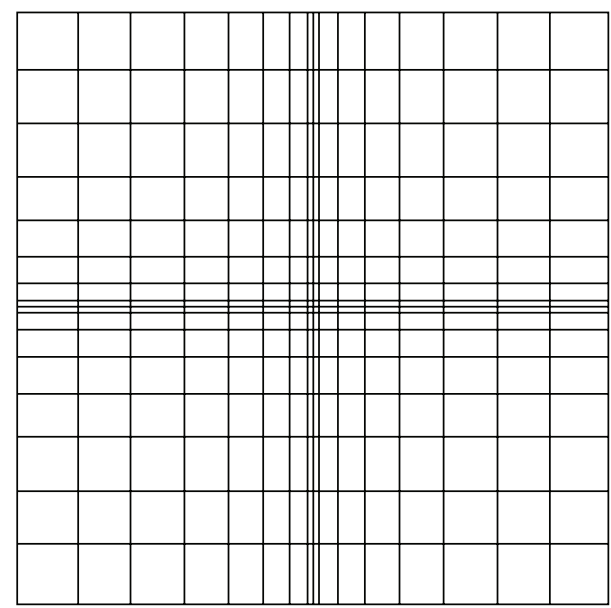

(b)

Figure 3: Mesh fashions: mesh 1 (a) and mesh 2 (b).

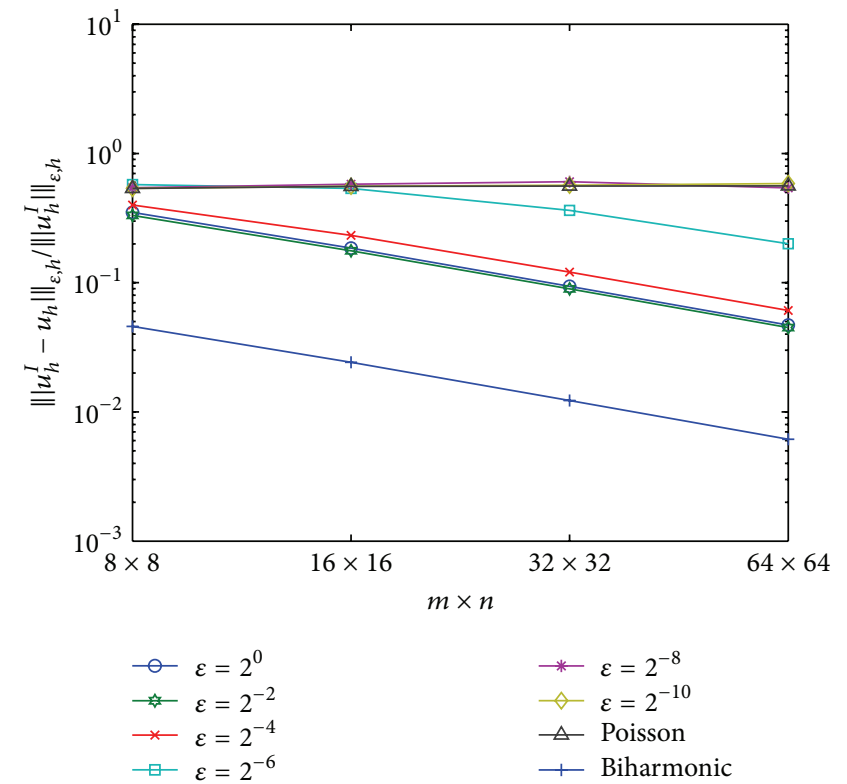

(a)

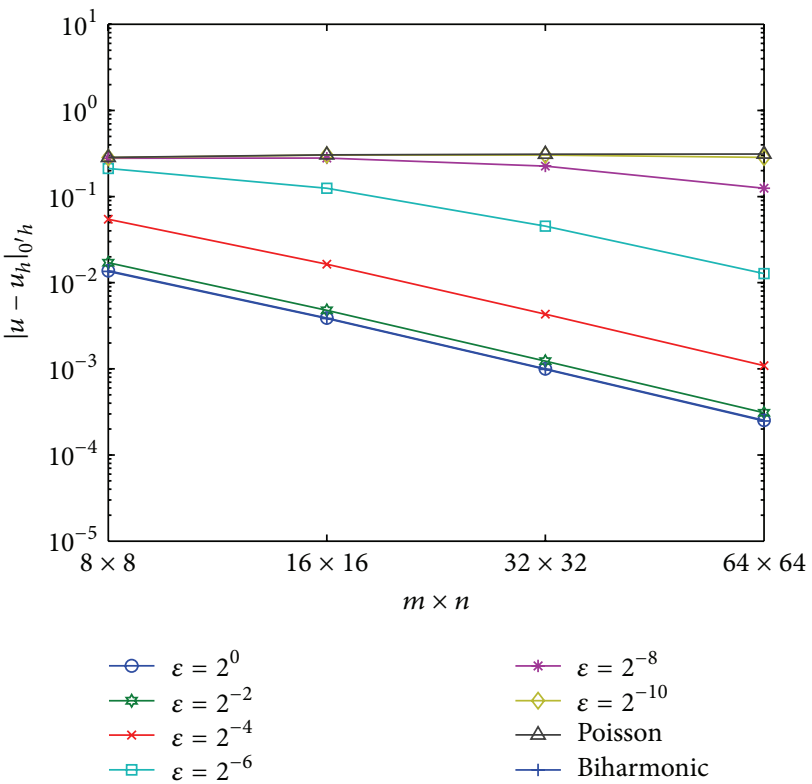

(b)

FiguRE 4: The errors $\left\|\left|u-u_{h}\right|\right\|_{\varepsilon, h} /\||u|\|_{\varepsilon, h}$ and $\left\|u-u_{h}\right\|_{0}$ under mesh 1 in the formulation (12).

TABLE 1: The errors $\left|\left\|u-u_{h}\right\|\right|_{\varepsilon, h} /|\|u\||_{\varepsilon, h}$ employing approximation form (12) under mesh 1.

\begin{tabular}{lcccr}
\hline$\varepsilon \backslash m \times n$ & $8 \times 8$ & $16 \times 16$ & $32 \times 32$ & $64 \times 64$ \\
\hline $2^{0}$ & $3.5080 e-001$ & $1.8514 e-001$ & $9.3821 e-002$ & $4.7068 e-002$ \\
$2^{-2}$ & $3.3197 e-001$ & $1.7650 e-001$ & $8.9611 e-002$ & $4.4977 e-002$ \\
$2^{-4}$ & $3.9897 e-001$ & $2.3228 e-001$ & $1.2090 e-001$ & $6.1071 e-002$ \\
$2^{-6}$ & $5.7470 e-001$ & $5.3768 e-001$ & $3.6322 e-001$ & $2.0029 e-001$ \\
$2^{-8}$ & $5.4334 e-001$ & $5.7884 e-001$ & $6.0446 e-001$ & $5.4125 e-001$ \\
$2^{-10}$ & $5.3774 e-001$ & $5.5800 e-001$ & $5.6811 e-001$ & $5.8575 e-001$ \\
\hline Poisson & $5.3734 e-001$ & $5.5616 e-001$ & $5.6087 e-001$ & $5.6205 e-001$ \\
\hline Biharmonic & $4.5852 e-002$ & $2.4188 e-002$ & $1.2255 e-002$ & $6.1481 e-003$ \\
\hline
\end{tabular}



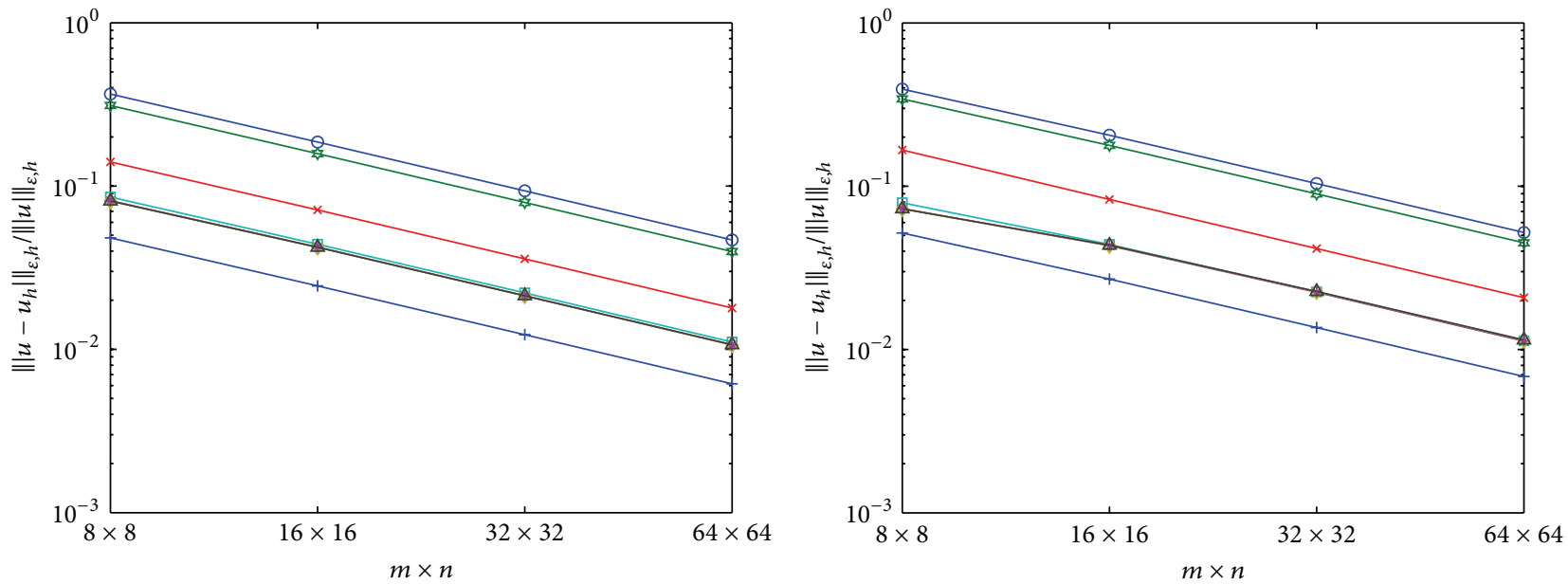

$\multimap \varepsilon=2^{0}$
$\rightarrow \varepsilon=2^{-2}$
$\nleftarrow \varepsilon=2^{-4}$
$\square \varepsilon=2^{-6}$

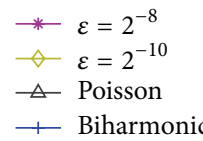

(a)

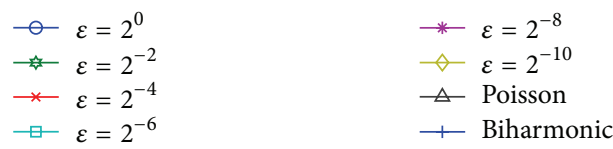

(b)

FIgURe 5: The errors $\left\|\left|u-u_{h}\right|\right\|_{\varepsilon, h} /\||u|\|_{\varepsilon, h}$ under mesh 1 (a) and mesh 2 (b).
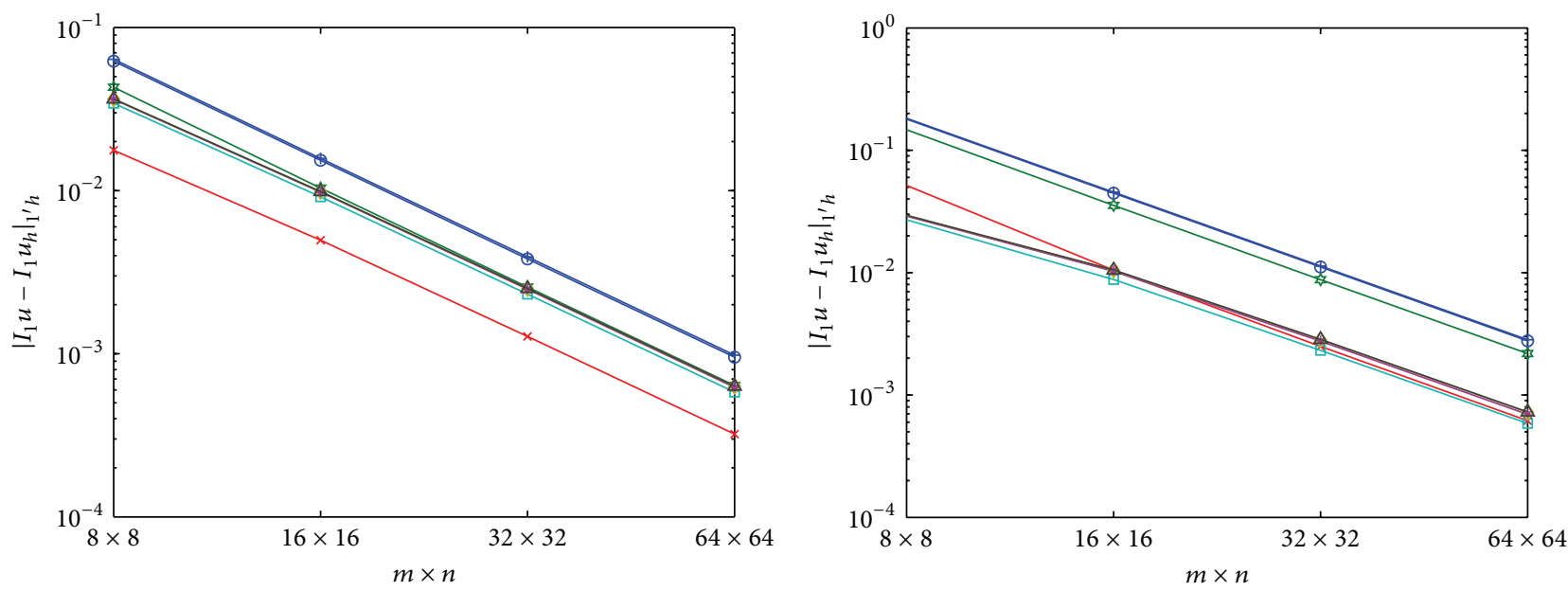

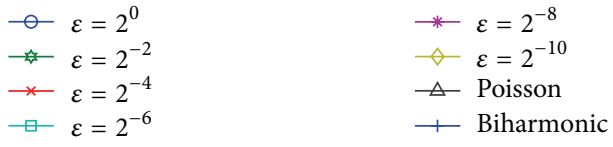

(a)

$$
\begin{aligned}
& -\varepsilon=2^{0} \\
& \nleftarrow \varepsilon=2^{-2} \\
& \rightarrow \varepsilon=2^{-4} \\
& \square \varepsilon=2^{-6}
\end{aligned}
$$

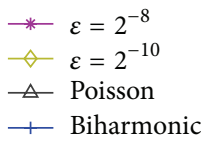

(b)

FIgURE 6: The error $\left|u-u_{h}\right|_{1}$ under mesh 1 (a) and mesh 2 (b).

finite element approximation, for different $\varepsilon$ and $h$. For a comparison, we also consider the case when $\varepsilon=0$, that is, the Poisson equation with Dirichlet boundary conditions, and the biharmonic problem

$$
\begin{gathered}
\Delta^{2} u=f, \quad \text { in } \Omega, \\
u=\frac{\partial u}{\partial n}=0, \quad \text { on } \partial \Omega,
\end{gathered}
$$

for this case, the corresponding relative error is presented by $\left|u-u_{h}\right|_{2, h} /|u|_{3}$. From Table 1 we can see that if the standard approximation scheme is applied, the method is divergent when $\varepsilon \rightarrow 0$.

We first present the relative errors in the energy norm $\left\|\left|u-u_{h}\right|\right\|_{\varepsilon, h} /\||u|\|_{\varepsilon, h}$ under mesh 1 when we use the standard finite element approximation.

To see the numerical effect more clearly, we plot the errors $\left\|\left|u-u_{h}\right|\right\|_{\varepsilon, h} /\||u|\|_{\varepsilon, h}$ and $\left\|u-u_{h}\right\|_{0}$ under different meshes in 

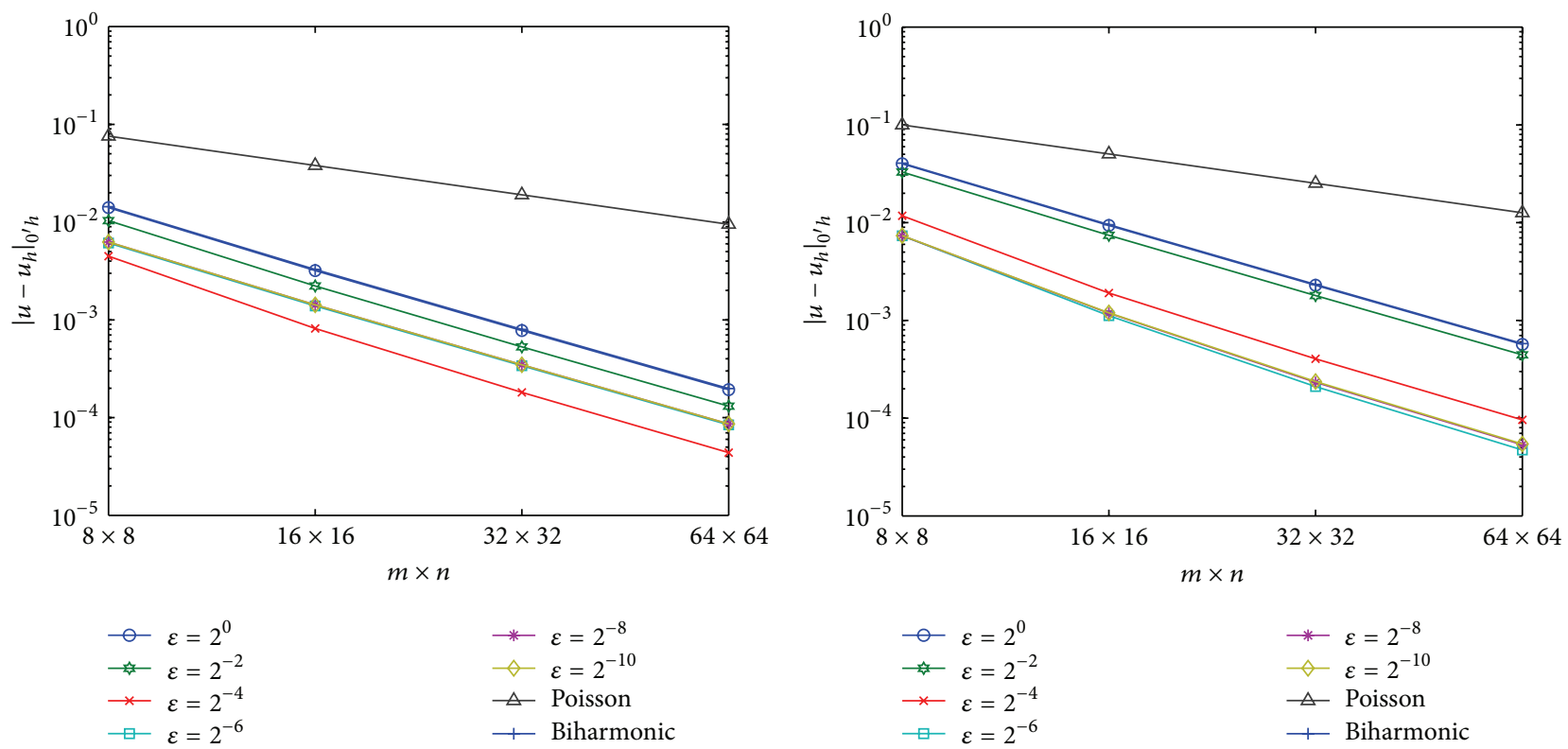

(a)

(b)

FIGURE 7: The error $\left|u-u_{h}\right|_{0}$ under mesh 1 (a) and mesh 2 (b).

TABLE 2: The errors $\left|\left\|u-u_{h}\right\|\right|_{\varepsilon, h} /|\|u\||_{\varepsilon, h}$ employing approximation form (14) under mesh 1.

\begin{tabular}{lcccr}
\hline$\varepsilon \backslash m \times n$ & $8 \times 8$ & $16 \times 16$ & $32 \times 32$ & $64 \times 64$ \\
\hline $2^{0}$ & $3.6561 e-001$ & $1.8595 e-001$ & $9.3374 e-002$ & $4.6737 e-002$ \\
$2^{-2}$ & $3.1147 e-001$ & $1.5806 e-001$ & $7.9321 e-002$ & $3.9697 e-002$ \\
$2^{-4}$ & $1.4057 e-001$ & $7.1356 e-002$ & $3.5816 e-002$ & $1.7925 e-002$ \\
$2^{-6}$ & $8.5681 e-002$ & $4.4048 e-002$ & $2.2146 e-002$ & $1.1088 e-002$ \\
$2^{-8}$ & $8.1275 e-002$ & $4.2229 e-002$ & $2.1271 e-002$ & $1.0642 e-002$ \\
$2^{-10}$ & $8.1006 e-002$ & $4.2163 e-002$ & $2.1281 e-002$ & $1.0662 e-002$ \\
\hline Poisson & $8.0988 e-002$ & $4.2159 e-002$ & $2.1284 e-002$ & $1.0667 e-002$ \\
\hline Biharmonic & $4.8120 e-002$ & $2.4478 e-002$ & $1.2292 e-002$ & $6.1526 e-003$ \\
\hline
\end{tabular}

TABLE 3: The errors $\left|\left\|u-u_{h}\right\|\right|_{\varepsilon, h} /|\|u\||_{\varepsilon, h}$ employing approximation form (14) under mesh 2.

\begin{tabular}{lcccc}
\hline$\varepsilon \backslash m \times n$ & $8 \times 8$ & $16 \times 16$ & $32 \times 32$ & $64 \times 64$ \\
\hline $2^{0}$ & $3.9326 e-001$ & $2.0520 e-001$ & $1.0374 e-001$ & $5.2017 e-002$ \\
$2^{-2}$ & $3.4184 e-001$ & $1.7775 e-001$ & $8.9771 e-002$ & $4.4999 e-002$ \\
$2^{-4}$ & $1.6659 e-001$ & $8.3029 e-002$ & $4.1538 e-002$ & $2.0775 e-002$ \\
$2^{-6}$ & $7.9112 e-002$ & $4.4059 e-002$ & $2.2540 e-002$ & $1.1326 e-002$ \\
$2^{-8}$ & $7.2810 e-002$ & $4.3249 e-002$ & $2.2399 e-002$ & $1.1253 e-002$ \\
$2^{-10}$ & $7.2776 e-002$ & $4.3471 e-002$ & $2.2618 e-002$ & $1.1408 e-002$ \\
\hline Poisson & $7.2784 e-002$ & $4.3503 e-002$ & $2.2652 e-002$ & $1.1438 e-002$ \\
\hline Biharmonic & $5.1683 e-002$ & $2.6973 e-002$ & $1.3637 e-002$ & $6.8377 e-003$ \\
\hline
\end{tabular}

a logarithm scale in Figure 4. Obviously the slope of the curve represents the convergence rate. We immediately get from the figures that when $\varepsilon<2^{-8}$, the errors are no longer descending, which means that the method is divergent. We should point out that, for biharmonic equation, the method is very efficient.
In Tables 2 and 3, we list the relative errors $\left\|\left|u-u_{h}\right|\right\|_{\varepsilon, h} /$ $\||u|\|_{\varepsilon, h}$ and the $L^{2}$ errors $\left\|u-u_{h}\right\|_{0}$ for the approximation scheme (14) under different meshes. In this case, for biharmonic problem, $u_{h} \in V_{h}$ is the solution of problem

$$
a_{h}\left(u_{h}, v_{h}\right)=\left(f, \Pi_{h}^{1} v_{h}\right), \quad \forall v_{h} \in V_{h} .
$$


When the formulation (14) is employed, the result shows that the method is uniformly convergent with respect to the parameter $\varepsilon$. Moreover, we can get that

$$
\lim _{\varepsilon \rightarrow 0} \frac{\left\|u-u_{h} \mid\right\|_{\varepsilon, h}}{\||u|\|_{\varepsilon, h}}=\frac{\left\|u-u_{h} \mid\right\|_{0, h}}{\|\mid u\|_{0, h}} .
$$

At the same time, we plot the logarithm figures of the errors under different meshes in Figure 5. The results consist with our analysis.

From Figures 5, 6, and 7, we can also see that, with the numerical results derived under mesh 1 and mesh 2 differ slightly, the method under mesh 2 is still very efficient when the approximation scheme (14) is employed. This matches our theoretical analysis.

\section{Conflict of Interests}

The authors declare that there is no conflict of interests regarding the publication of this paper.

\section{Acknowledgments}

The work is supported by the National Natural Sciences Foundation of China (no. 11126272 and 11201122), High-Level Personal Foundation of Henan University of Technology (Grant no. 2009BS066), and The basic and frontier project of Henan province (no. 132300410232).

\section{References}

[1] S. C. Chen, Y. C. Zhao, and D. Y. Shi, "Non $C^{0}$ nonconforming elements for elliptic fourth order singular perturbation problem," Journal of Computational Mathematics, vol. 23, no. 2, pp. 185-198, 2005.

[2] M. Wang, J. C. Xu, and Y. C. Hu, "Modified Morley element method for a fourth order elliptic singular perturbation problem," Journal of Computational Mathematics, vol. 24, no. 2, pp. 113-120, 2006.

[3] T. K. Nilssen, X. C. Tai, and R. Winther, "A robust nonconforming $H^{2}$-element," Mathematics of Computation, vol. 70, no. 234, pp. 489-505, 2001.

[4] S. Zhang and M. Wang, "A posteriori estimator of nonconforming finite element method for fourth order elliptic perturbation problems," Journal of Computational Mathematics, vol. 26, no. 4, pp. 554-577, 2008.

[5] B. Semper, "Conforming finite element approximations for a fourth-order singular perturbation problem," SIAM Journal on Numerical Analysis, vol. 29, no. 4, pp. 1043-1058, 1992.

[6] J. A. Nitsche and A. H. Schatz, "Interior estimates for RitzGalerkin methods," Mathematics of Computation, vol. 28, pp. 937-958, 1974.

[7] A. H. Schatz and L. B. Wahlbin, "On the finite element method for singularly perturbed reaction-diffusion problems in two and one dimensions," Mathematics of Computation, vol. 40, no. 161, pp. 47-89, 1983.

[8] G. Strang, "Variational crimes in the finite element method," in The Mathematical Foundations of the Finite Element Method with Applications to Partial Differential Equations, A. R. Aziz, Ed., pp. 689-710, Academic Press, New York, NY, USA, 1972.
[9] F. Stummel, "The generalized patch test," SIAM Journal on Numerical Analysis, vol. 16, no. 3, pp. 449-471, 1979.

[10] Z. C. Shi, "The F-E-M test for convergence of nonconforming finite elements," Mathematics of Computation, vol. 49, no. 180, pp. 391-405, 1987.

[11] S. C. Chen, D. Y. Shi, and H. Ichiro, "Generalized estimate formulation of nonconforming finite elements," Mathematica Numerica Sinica, vol. 22, no. 3, pp. 295-300, 2000.

[12] Z. C. Shi, "On the convergence of the incomplete biquadratic nonconforming plate element," Mathematica Numerica Sinica, vol. 8, no. 1, pp. 53-62, 1986.

[13] Z. C. Shi, "The generalized patch test for Zienkiewicz's triangles," Journal of Computational Mathematics, vol. 2, no. 3, pp. 279-286, 1984.

[14] Z. C. Shi and S. C. Chen, "An analysis of a nine-parameter plate element of Specht," Mathematica Numerica Sinica, vol. 11, no. 3, pp. 312-318, 1989.

[15] D. Y. Shi, S. P. Mao, and S. C. Chen, "On the anisotropic accuracy analysis of ACM's nonconforming finite element," Journal of Computational Mathematics, vol. 23, no. 6, pp. 635-646, 2005.

[16] S. P. Mao and S. C. Chen, "Convergence analysis of Morley element on anisotropic meshes," Journal of Computational Mathematics, vol. 24, no. 2, pp. 169-180, 2006.

[17] Z. C. Shi and Q. Y. Chen, "A new high accuracy rectangular element," Scince in China A, vol. 30, no. 6, pp. 504-515, 2000.

[18] L. S. D. Morley, "The triangular equilibrium element in the solution of plate bending problems," Aero Quartet, vol. 19, pp. 149-169, 1968.

[19] P. Lascaux and P. Lesaint, "Some nonconforming finite elements for the plate bending problem," Analyse Numérique, vol. 9, no. 1, pp. 9-53, 1975.

[20] Z. C. Shi, "Error estimates for the Morley element," Mathematica Numerica Sinica, vol. 12, no. 2, pp. 113-118, 1990.

[21] S. P. Mao and Z. C. Shi, "High accuracy analysis of two nonconforming plate elements," Numerische Mathematik, vol. 111, no. 3, pp. 407-443, 2009.

[22] M. Wang, "On the necessity and sufficiency of the patch test for convergence of nonconforming finite elements," SIAM Journal on Numerical Analysis, vol. 39, no. 2, pp. 363-384, 2001.

[23] P. G. Ciarlet, The Finite Element Method for Elliptic Problems, North-Holland, Amsterdam, The Netherlands, 1978.

[24] H. Q. Zhang and M. Wang, The Mathematical Theory of Finite Elements, Science Press, Beijing, China, 1991.

[25] T. Apel and M. Dobrowolski, "Anisotropic interpolation with applications to the finite element method," Computing, vol. 47, no. 3-4, pp. 277-293, 1992.

[26] S. C. Chen, D. Y. Shi, and Y. C. Zhao, "Anisotropic interpolation and quasi-Wilson element for narrow quadrilateral meshes," IMA Journal of Numerical Analysis, vol. 24, no. 1, pp. 77-95, 2004.

[27] P. L. Xie, Nonconforming finite element methods for fourth order elliptic problems [Ph.D. thesis], Fudan University, Shanghai, China, 2009. 


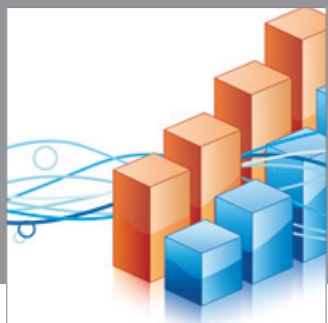

Advances in

Operations Research

mansans

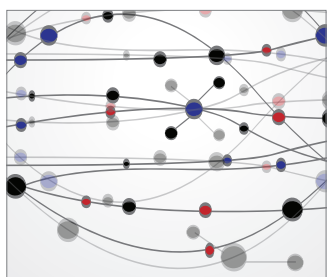

The Scientific World Journal
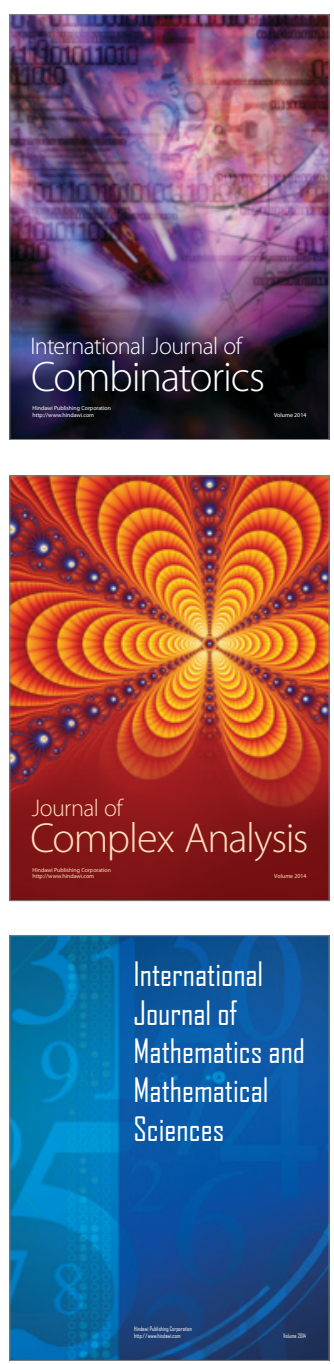
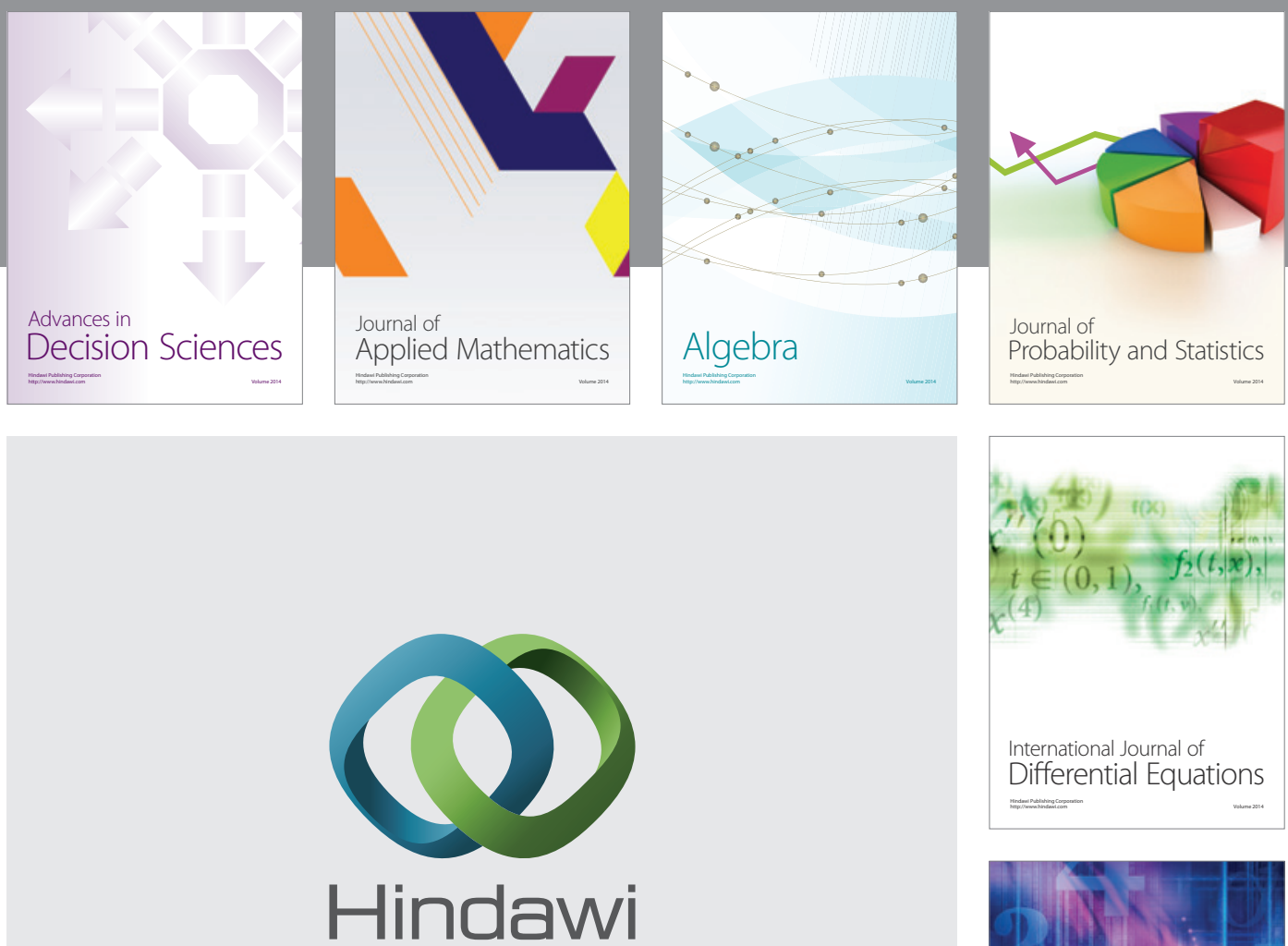

Submit your manuscripts at http://www.hindawi.com
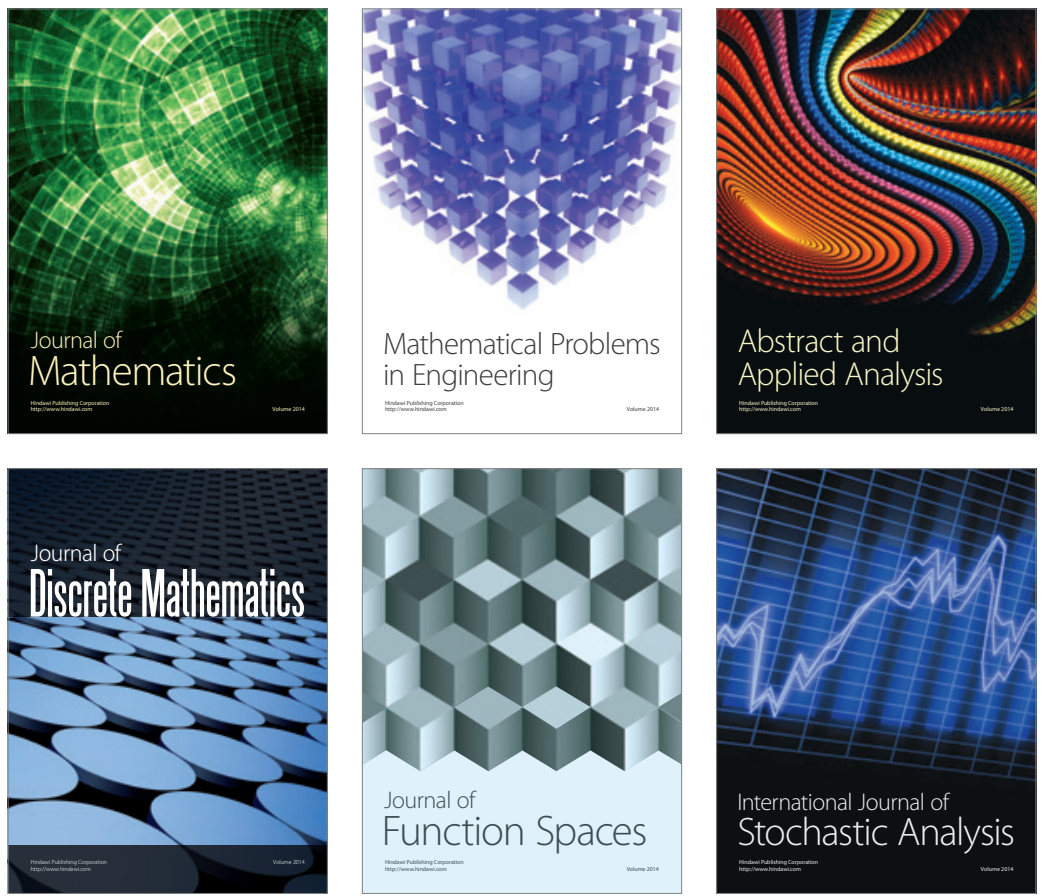

Journal of

Function Spaces

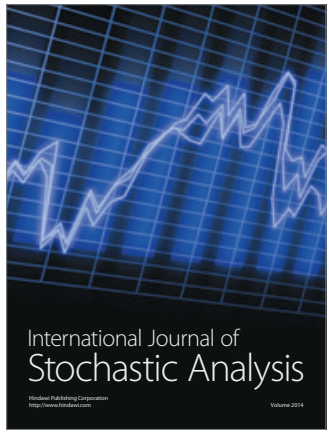

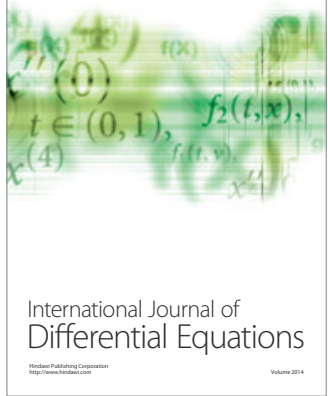
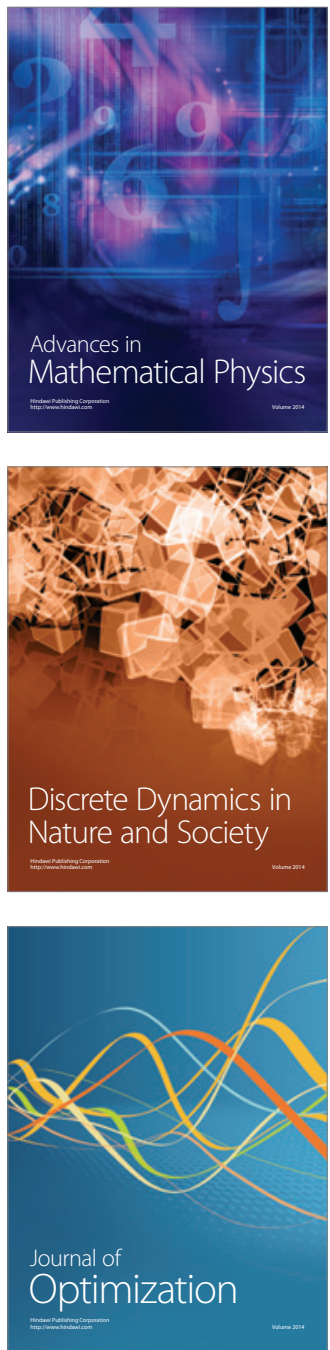